\title{
RISK-INFORMED PROCESS AND TOOLS FOR PERMITTING HYDROGEN FUELING STATIONS
}

\author{
Jeffrey LaChance ${ }^{1}$, Andrei Tchouvelev ${ }^{2}, \mathrm{Jim}_{\mathrm{Ohi}}{ }^{3}$ \\ ${ }^{1}$ Sandia National Laboratories, P.O. Box 5800, Albuquerque, NM, 87104, USA, \\ jllacha@sandia.gov \\ ${ }^{2}$ AVT \& Associates, 6591 Spinnaker Circle, Mississauga, ON, L5W 1R2, Canada, \\ atchouvelev@tchouvelev.org \\ ${ }^{3}$ National Renewable Energy Laboratory, 1617 Cole Blvd., Golden, CO 80401, USA \\ jim_ohi@nrel.gov
}

\begin{abstract}
The permitting process for hydrogen fueling stations varies from country to country. However, a common step in the permitting process is the demonstration that the proposed fueling station meets certain safety requirements. Currently, many permitting authorities rely on compliance with wellknown codes and standards as a means to permit a facility. Current codes and standards for hydrogen facilities require certain safety features, specify equipment made of material suitable for hydrogen environment, and includeseparation or safety distances. Thus, compliance with the code and standard requirements is widely accepted as evidence of a safe design. However, to ensure that a hydrogen facility is indeed safe, the code and standard requirements should be identified using a risk-informed process that utilizes an acceptable level of risk. When compliance with one or more code or standard requirements is not possible, an evaluation of the risk associated with the exemptions to the requirements should be understood and conveyed to the Authority Having Jurisdiction (AHJ). Establishment of a consistent risk assessment toolset and associated data is essential to performing these risk evaluations. This paper describes an approach for risk-informing the permitting process for hydrogen fueling stations that relies primarily on the establishment of risk-informed codes and standards. The proposed risk-informed process begins with the establishment of acceptable risk criteria associated with the operation of hydrogen fueling stations. Using accepted Quantitative Risk Assessment (QRA) techniques and the established risk criteria, the minimum code and standard requirements necessary to ensure the safe operation of hydrogen facilities can be identified. Riskinformed permitting processes exist in some countries and are being developed in others. To facilitate consistent risk-informed approaches, the participants in the International Energy Agency (IEA) Task 19 on hydrogen safety are working to identify acceptable risk criteria, QRA models, and supporting data ${ }^{1}$.
\end{abstract}

\footnotetext{
${ }^{1}$ This work is being conducted as part of Task 19 - Hydrogen Safety, a collaboration of experts from eight countries, under the Hydrogen Implementing Agreement of the International Energy Agency which operates under an international agreement of more than 26 countries. The overall goal of the IEA task on hydrogen safety is to develop data and other information that will facilitate the accelerated adoption of hydrogen systems and supports the accomplishment of the Hydrogen Implementing Agreement's stated mission: “...to accelerate hydrogen implementation and widespread utilization." Because of the nature of the International Energy Agency as an international agreement between governments, it is hoped that such collaboration will complement other efforts to build the technology base around which codes and standards can be developed.
} 


\section{INTRODUCTION}

It is common knowledge that emerging hydrogen fueling stations are being permitted and approved based on existing regulations, codes and standards (RC\&S) \& well as other requirements that vary from jurisdiction to jurisdiction. These "other requirements" may include some sort of a risk assessment that aims to demonstrate to regulatory authorities that adequate risk mitigation measures are in place and adequate safety is provided. Once the permission to operate a station is granted, it is presumed that the station satisfies necessary safety requirements (at least those that are imposed by a local AHJ). To minimize and even eliminate these "other requirements" and make the permitting and approval process consistent and harmonized, hydrogen specific RC\&S need to contain requirements that, if followed, would ensure compliance with acceptable levels of risk or in other words be riskinformed. To better understand the background for the development of risk-informed RC\&S there is a need to review the relationship between "safety" and "risk".

\section{SAFETY AND RISK}

Paradigm 1: Safety is freedom from unacceptable risk [1]. This internationally accepted paradigm effectively means that:

- Risk is the measure of safety

- Society accepts the fact that there is neither absolute (i.e., 100\%) safety nor zero risk

- Society, de facto, establishes acceptable levels of risk

Paradigm 2 Risk criteria are the terms of reference by which the sig nificance of risk is assessed [2]. This internationally accepted paradigm effectively means that:

- Society, de facto, establishes terms of reference for acceptable levels of risk or risk acceptance criteria

One can then go further and suggest that should the world share the same risk acceptance criteria, then any product acceptance from a safety perspective would be much easier: just prove the compliance of your product to acceptable risk levels in your home country and ship it anywhere around the world.

Paradigm 3: No new technology shall impose a greater societal risk than an incumbent similar technology; in other words, they have to be at least at par with each other in terms of societal risk (i.e., they must satisfy the same risk acceptance criteria). Although this paradigm is not written in any ISO / IEC guidelines, it is de facto being used across the developed world.

Application of these three paradigms to emerging hydrogen energy and technology applications in general and hydrogen fueling infrastructure in particular is the main theme of the IEA Task 19 Hydrogen Safety. It was unanimously accepted by the expert participants of the IEA Task 19 Hydrogen Safety that the hydrogen fueling infrastructure must be at least at par with the existing fueling infrastructure in terms of societal risk.

The necessity to comply with risk acceptance criteria suggests three important things:

1. Any product must have a basic design that satisfies risk acceptance criteria and thus ensures a minimum acceptable level of safety under intended operating conditions.

2. Methods and tools are required to measure and verify product compliance with acceptable levels of risk.

3. Codes and standards that identify minimum design, performance and installation requirements as well as regulations that guide permitting and approval processes have to reflect those risk acceptance criteria in order to become risk-informed. 
That is why it was also accepted by the IEA Task 19 Hydrogen Safety group that it is paramount to determine appropriate risk acceptance criteria that ensure acceptable safety levels for the emerging hydrogen fueling infrastructure.

\subsection{RISK ACCEPTANCE CRITERIA AND RISK-INFORMED RC\&S}

Establishment of risk criteria is a key element required to utilize a risk-informed approach. Since the primary concern is the potential for personnel injury, risk criteria can be established for all the people exposed to the consequences of facility-related accidents, which could include the public located outside the boundaries of the facility, users of the facility, and the facility workers. Societal or public risk is generally the main focus in risk assessments. In most QRA applications, the risk levels for the public are generally set one to two orders of magnitude less than the level for workers. Depending on the accident consequence, the selected risk criteria could reflect acceptance levels for either injuries or fatalities.

Risk criteria can be specified with regard to individuals or the society at large. Individual risk reflects the frequency that an average person located permanently at a certain location is harmed. Characterization of the population surrounding a facility is thus not required to evaluate individual risk. Societal risk reflects the relationship between the frequency and the number of people harmed. Evaluation of societal risk requires determination of the population surrounding a facility. For the application of QRA to determine the separation distances specified in codes and standards, the use of individual risk measures $m$ ay be the most appropriate since they are site independent.

Risk acceptance criteria for both individual and societal risk, though de facto exist everywhere, are not always obvious. In some world jurisdictions, like in most Western European countries and Australia, they are incorporated into law. In the U.S. and Canada, to the contrary, as in many other jurisdictions around the world, they are not defined in any way and are, thus, subject to interpretation.

Selection of individual risk criteria should be based on sound arguments and reflect the consensus of all stakeholders. Ideally, the risk associated with the widespread development of hydrogen refueling stations should not substantially increase the injury or fatality risk of an individual. As mentioned in the previous section, this concept is not new and in fact has been utilized in the nuclear power industry. A critical question is what level of risk should be utilized in this concept? Several options are discussed here for consideration by codes and standards groups and other decision makers.

The first is to specify that the risk from hydrogen accidents be some fraction of the total risk to individuals from all unintentional injuries. This approach has been adopted by the U.S. Nuclear Regulatory Commission (NRC) in their efforts to risk-inform the regulations for nuclear power plants. The NRC risk criteria is based on the principle that the risk to an average individual in the vicinity of a nuclear power plant should be a fraction $(0.1 \%)$ of the sum of the fatality risk resulting from other accidents to which members of the public are generally exposed in everyday life (e.g., fatal automobile accidents). At the time the NRC established this policy in 1995, the individual fatality risk in the U.S. was approximately $5 \times 10^{-4} / \mathrm{yr}$. Recent data [3] suggest that the individual fatality risk from unintentional injuries in the United States is on the order of $3.8 \times 10^{-4} / \mathrm{yr}$. The individual injury frequency from unintentional accidents is approximately $0.09 / \mathrm{yr}$.

For comparison, the individual fatality risk from various causes in the Netherlands [4] is reported to be $2 \times 10^{-3} / \mathrm{yr}$ from smoking, $4 \times 10^{-3} / \mathrm{yr}$ from traffic accidents, $1 \times 10^{-2} / \mathrm{yr}$ from all diseases, and $5 \times 10^{-4} / \mathrm{yr}$ from natural radiation.

The European Industrial Gas Association (EIGA) in its document Determination of Safety Distances [5] proposes to use as the basis for selection of a fatality risk criteria the least exposed category children, who between the ages of 5 and 15 have he lowest natural fatality risk. The document suggests $2 \times 10^{-4}$ per annum as an average minimum natural individual fatality risk for a westernized, (European) industrialized, population. This number includes all possible harm exposures in occupational, traffic, and home/leisure segments, with approximately $0.7 \times 10^{-4}$ per annum 
contribution of each major segment. Applying the EIGA logic to refueling stations we may suggest that since the minimum natural individual fatality risk for the "traffic" segment is $0.7 \times 10^{-4}$ per annum, then the risk from refueling should be at least half of that, i.e., $3.5 \times 10^{-5}$ per annum. This means that recommended individual risk criteria for hydrogen refueling will constitute approximately $1 / 6$ of natural individual fatality risk.

The selection of the fractional increase in these individual risk values that would be used in establishing hydrogen-related risk criteria is a critical decision that regulators and other policy makers will have to make if this approach is utilized. As can be seen from the comparison of the above examples, the EIGA has suggested using a higher percentage $(17.5 \%)$ of the existing fatality frequency $\left(2 \times 10^{-4} / \mathrm{yr}\right)$ than used by the NRC as a criterion to measure harm to the public (i.e., an individual fatality risk $=3.5 \times 10^{-5} / \mathrm{yr}$ ). The EIGA also have suggested using a criterion for "no harm" to the public that is two orders of magnitude greater than the harm criterion. The European Integrated Hydrogen Project (EIHP2) also utilized this approach based on an average fatality death rate of $2 \times 10^{4} / \mathrm{yr}$ and a specification that the individual fatality risk for hydrogen refueling stations be $1 \%$ of this value [6]. The EIHP has also established individual fatality risk value of $1 \times 10^{-4} / \mathrm{yr}$ for refueling station workers.

A variation of this first option is to utilize just the individual fatality and injury risk associated with only fires and explosions. Considering that these are the major concerns associated with hydrogen facility operation, this may be a better approach but it requires careful consideration of available fire and explosion statistics. The individual fatality risk due to fires in the United States is $1.2 \times 10^{-5} / \mathrm{yr}$ and the corresponding value for explosions and overpressure events is $6.0 \times 10^{-7} / \mathrm{yr}$ B]. Further examination of this data indicates that the individual fatality risk from fires involving highly flammable materials such as hydrogen is approximately $2.0 \times 10^{-7} / \mathrm{yr}$, the risk from structure fires is $9.5 \times 10^{-6} / \mathrm{yr}$, the risk from fires outside of structures is $8 \times 10^{-8} / \mathrm{yr}$, and the risk from unspecified fire sources is $1.1 \times 10^{-6} / \mathrm{yr}$. Data on fire-related injuries have not been identified, but the individual injury risk from fires is expected to be approximately two orders of magnitude greater than the values cited above.

A better option, as mentioned above, is to specify that the risk associated with hydrogen refueling stations be at par with the risk associated with gasoline or compressed natural gas (CNG) stations. Unfortunately, no published risk assessments for either gasoline or CNG refueling stations that could provide those risk estimates have been identified. However, there are some limited data on the frequency of fires in public gasoline stations [7] for the five-year period of 19941998 (no published data for CNG stations were identified) that could be used to establish such a comparative criterion. This data indicate that the average frequency of a fire at a gasoline station is approximately $7.4 \times 10^{-2} / \mathrm{yr}$. A majority of the reported fires were initiated by vehicle fires, and only a small fraction $(\sim 4 \%)$ was related to spills of gasoline leading to fires or explosions. When vehicle fires are eliminated, the fire frequency is approximately $2.8 \times 10^{-2} / \mathrm{yr}$, and when only spills are considered, the average fire frequency is approximately $3 \times 10^{-3} / \mathrm{yr}$. The reported fires resulted in, on average, 2 deaths/yr and 70 injuries/yr. Since there were approximately 100,000 public service stations in operation during this period, the average frequencies of a fatality or injury associated with the operation of a single gasoline station are approximately $2 \times 10^{-5} / \mathrm{yr}$ and $7 \times 10^{-5} / \mathrm{yr}$, respectively. If vehicle fires are eliminated, the average fatality and injury frequencies associated with operation of an individual gasoline station are approximately $1 \times 10^{-5} / \mathrm{yr}$ and $3.3 \times 10^{-4} / \mathrm{yr}$, respectively. The corresponding fatality and injury frequencies attributable to gasoline spills are approximately $5 \times 10^{-6} / \mathrm{yr}$ and $9 \times 10^{-5} / \mathrm{yr}$.

An alternative (and, probably, the best) way to determine the risk associated with the existing gasoline infrastructure would be to conduct QRAs of a variety of existing gasoline stations using best available QRA and modeling tools and data. The same could be repeated for other existing types of fueling stations (e.g., compressed natural gas) as well as for those with co-located fuels. That, however, would require full cooperation of station owners, which is hard if not impossible to achieve. There is no interest from their side to potentially expose themselves to additional liability due to "surprises" that 
might be uncovered in the course of such a rigorous analysis, which otherwise stay hidden within the grandfathered permitting and approval process, which is based on non-risk-informed RC\&S.

Harm scale aversion will have to be taken into account when estab lishing societal risk criteria. Recent studies surveyed by HSE [8] earlier this year clearly suggest that society in general is more willing to accept small but frequent accidents rather than a single accident with large consequences, although the resulting number of casualties would be equal in both cases. In the Netherlands, for example, the probability of 10 fatalities in a single incident can be at most once in 100,000 years for installations that include "hazardous companies" such as large petrochemical plants, LPG filling stations and hazardous material storage facilities. An incident involving 100 fatalities may at most occur once in 10 million years [9]. As can be seen, the aversion slope in this example is -2, i.e. for every order of magnitude increase in number of fatalities, the probability must drop by two orders of magnitude. As was stated in the EIHP2 report [6], "The slope of the FN curve is designed to reflect the society's aversion to single accidents with multiple fatalities as opposed to several accidents with few fatalities". It is important to note, however, that though in the Netherlands individual (location-based) risk criterion (set at $1 \times 10^{-6} / \mathrm{yr}$ ) acquired legal status in July 2004 and is part of the External Safety (Installation) Decree, the societal risk criteria quoted above are not legalized and still remain as guid ance values [9]. This is probably an indication that the individual risk should likely be the main focus of risk-informed codes and standards, while the societal risk will remain the focus of site QRAs for the foreseeable future.

IEA Task 19 within its subtask on risk management intends to perform detailed analysis of similar data available within IEA countries and recommend uniform risk acceptance criteria for hydrogen refueling infrastructure for implementation globally.

Once acceptable levels of risk are determined they can be incorporated into hydrogen codes and standards, thus making them risk-informed. Compliance with risk-informed codes and standards would mean that station designs meeting their requirements automatically meet acceptable levels of safety and can be permitted without additional requirements.

\subsection{RISK-INFORMED PROCESS FOR DEVELOPMENT OF HYDROGEN CODES AND STANDARDS}

Hydrogen fueling stations in many countries including the United States are permitted by one or more AHJs which generally include a local government entity. A critical step in the permitting process is the demonstration that the proposed fueling station meets the AHJ safety requirements. Currently, many AHJs rely on compliance with well-known codes and standards as one means to license a facility. Thus, the establishment of code requirements is critical to ensuring the safety of these facilities.

Codes and standards applicable to hydrogen facilities are currently developed by a variety of standards and code development organizations (SDOs) in the United States including the National Fire Protection Association (NFPA), American Society of Mechanical Engineers (ASME), Compressed Gas Association (CGA), CSA America, Underwriters Laboratories (UL), and the International Code Council (ICC). The current codes and standards for hydrogen facilities specify that the facilities have certain safety features, use equipment made of material suitable for a hydrogen environment, and have specified separation distances. Codes and standards are typically adopted by governmental authorities, in which case they become regulations, and compliance with them is widely accepted as evidence of a safe design.

The bases for the current hydrogen codes and standards are not well documented and thus the safety of facilities complying with them may be questioned by some. For the most part, SDOs in the past have relied upon expert panels to establish necessary code requirements. The bases for the expert judgements are not documented but likely reflect a combination of good engineering practices to address the potential hazards associated with hydrogen, historical precedence based on requirements for other fuels such as $\mathrm{CNG}$, and anecdotal knowledge of past problems in hydrogen facilities. It is 
possible that some of the requirements are based on experimental or deterministic analyses of selected accidents that were felt to represent credible, but not worst case, accidents. It should be noted that this process appears to have worked to ensure safety in many industries primarily through the continuing process of adapting the applicable code requirements to address issues identified from an analysis of accidents.

Several of the SDOs are working to modify existing codes and standards and draft new ones related to the use of hydrogen as an automobile fuel and also for other electrical generation applications (i.e., stationary fuel cell applications). These efforts include re-establishing the bases for some code requirements such as separation distances. For example, the NFPA has launched an effort to compile all requirements for hydrogen applications into one model code, NFPA 2, Hydrogen Technologies. The Technical Committee for NFPA 2 is systematically reviewing all NFPA model codes and standards related to hydrogen as part of this compilation. In addition, the Committee has created a number of task groups to assess the technical foundations of requirements in these codes and standards. One of these task groups is examining the technical bases for separation distances and is applying recent research on hydrogen behavior and QRA techniques as part of this examination.

In addition, several groups have been established to coordinate the development and implementation of a consistent set of hydrogen-related codes and standards. Three examples in the U.S. are the National Hydrogen and Fuel Cells Codes and Standards Coordinating Committee, managed by the U.S. Department of Energy, the National Hydrogen Association, and the U.S. Fuel Cell Council, and the independent Hydrogen Industry Panel on Codes (HIPOC), which focuses on harmonizing requirements in NFPA an ICC model codes. In addition, the U.S. FreedomCAR and Fuel Partnership have created a Codes and Standards Technical Team to coordinate the research and development needed to establish a scientific foundation for requirements in hydrogen and fuel codes and standards. The Codes and Standards Working Group of the Canadian Transportation Fuel Cell Alliance (CTFCA) has a similar charter in Canada. A common theme in the efforts of the SDOs and industry cooperation groups is the establishment of science-based codes and standards that will ensure public safety from the use of hydrogen as a fuel. The use of a risk-informed process is one way to establish the requirements in these new codes and standards necessary to ensure public safety.

A risk-informed process utilizes risk insights obtained from QRAs combined with other considerations to establish code requirements. The QRAs are used to identify and quantify scenarios for the unintended release of hydrogen, identify the significant risk contributors at different types of hydrogen facilities, and to identify potential accident prevention and mitigation strategies to reduce the risk to acceptable levels. Examples of other considerations used in this risk-informed process can include the results of deterministic analyses of selected accidents scenarios, the need for defense-in-depth for certain safety features (e.g., overpressure protection), the use of safety margins in the design of highpressure components, and requirements identified from the actual occurrences at hydrogen facilities. A key component of this process is that both accident prevention and mitigation features are included in the code and standard requirements.

A risk-informed process can help establish the baseline design and operational requirements for hydrogen fueling stations. Although separation distances are a key safety parameter specified in hydrogen codes and standards, there are other design and operational requirements that are used to ensure safe operation. Key design features currently specified in hydrogen codes and standards include interlocked leak detection and isolation capability, dilution ventilation, emergency venting, emergency manual shutoff switches, pressure relief de vices and associated vent lines, process monitoring and safety interlocks, and fail safe design requirements (e.g., closure of isolation valves on loss of power). Operational requirements can include normal operating procedures, maintenance and surveillance procedures, limiting conditions of operation, and emergency procedures in the case of major accidents.

QRA can be used in several different ways to help establish the code and standard requirements for hydrogen facilities. First, by analyzing a complete set of possible accidents, the risk drivers for these facilities can be identified. Accident prevention and mitigation features can then be specified to 
address all accidents. The number or type of specified requirements can be determined by both the cause and frequency of the accident and the associated risk. For example, one could specify that redundant or highly reliable design features be in place to address commonly occurring events such as small hydrogen leaks and that procedural requirements include inspections and emergency response plans to prevent or respond to more catastrophic accidents such as tank ruptures. The risk-reduction potential of the specified design and operational features can then be evaluated using the QRA models. Important questions that can be answered using QRA include identifying which of these design and operational features are most effective to reduce risk and determining whether additional features are necessary to achieve an acceptable level of risk.

The requirements for safe operation are dependent upon the type of facility. For example, the different hydrogen generation methods (e.g., liquid hydrogen vaporization, steam reforming of natural gas, or electrolysis) will introduce unique risk scenarios that have to be addressed by specific code requirements applicable to those facilities. QRAs of a full spectrum of facilities can be used to help identify their associated risk drivers and required prevention and mitigation features unique to specific types of facilities.

Similarly, some of the requirements in codes and standards are dependent upon facility parameters such as the volume and pressure of stored hydrogen. An example of this is the separation distances that are specified for hydrogen gas and liquid storag e areas. Currently, NFPA 55 [10] provides separation distances as a function of the total liquid and gaseous hydrogen volumes. The Canadian Hydrogen Installation Code provides separation distances as a function of the total mass for gaseous hydrogen and otal volume for liquid hydrogen. NFPA 52 [11] does not differentiate separation distances in this fashion. The most progressive approach so far in specifying separation distances has been applied by the ISO/TC 197 Working Group (WG) 11 in developing a Technical Specification for Hydrogen Refueling Stations [12]. In its recommendation, WG 11 has used a variety of combinations of stored mass, storage volume and pressure in defining separation distances. The ISO/TC 197 approach reflects the potential risks from storage systems that are driven either by storage pressure or stored mass, or both. Whether the parameters currently being used to differentiate separation distances in these codes are adequate for future hydrogen refueling stations and other hydrogen facilities is an issue that is being addressed by the SDOs. For example, higher gas storage and dispensing pressures than contemplated during the creation of the current codes are being considered for hydrogen refueling stations (gas storage cylinders with pressures of $70 \mathrm{MPa}$ or greater are being incorporated into facility designs). A consequence of these higher pressures is that the required separation distances may be significantly greater than currently specified.

QRAs can also evaluate the risk associated with selected accidents used in establishing code requirements. An example of this is the selection of a particular leak size to establish separation distances. Separation distances based on a probable leak diameter can introduce a high level of risk from leaks that are less probable but which result in high consequences. Thus, it is desirable that risk considerations be included in the determination of accidents.

\subsection{RISK-INFORMED FRAMEWORK FOR PERMITTING HYDROGEN FUELING STATIONS}

The challenge faced in permitting hydrogen fueling stations is to have a process that is relatively simple and fast but also ensures that the facilities are safe. To date, the experience in permitting hydrogen fueling stations is limited but growing. In many cases, the AHJs have applied the same approach used in the past to license other types of fueling stations - reliance upon conformance to applicable codes and standards. However, many of these hydrogen facilities are first-of-a-kind designs and consequently were subjected to more risk analysis in the permitting process.

The level of risk assessment that is currently performed for hydrogen fueling stations is variable ranging from purely qualitative assessments to QRAs. Designers of these facilities often only use qualitative techniques such as Failure Modes and Effects Analysis (FMEAs) to help identify the potential hazards and required prevention and mitigation features. Others also use semiquantitative 
risk matrices where different accidents are binned into quantitative frequency and consequence bins based on subjective judgements. If the associated risk of an accident is deemed high, a QRA of the accident scenario may be performed. Some AHJs require risk management plans that include emergency response procedures to address the high risk scenarios. Since the use of hydrogen as an automobile fuel is a relatively new and unproven technology, it seems prudent to perform more comprehensive QRAs for the early facilities to provide confidence that they are indeed safe.

Once the safety bases for hydrogen fueling facilities have been established through analysis that leads to the development of risk-informed codes and standards, risk analysis of fueling stations may not be required. At this point in time, the $\mathrm{AHJ}$ s can be assured that a facility designed, constructed, and operated according to code requirements should meet accepted risk criteria (assuming risk criteria are utilized in the code development process) and thus will not require additional risk analysis. In particular, standard designs that meet the code requirements should require very little deliberation by the AHJs. However, some facilities may require special design or operational considerations that may require exemptions from the codes and standard requirements. For example, $\dot{\mathbf{t}}$ is envisioned that many hydrogen fueling stations will be initially located in existing stations for other fuels such as gasoline and CNG. Thus, limited space will be available for locating the hydrogen-related generation, storage, and dispensing equipment, and the potential for additional accident initiators is introduced. Similarly, locations for new, dedicated hydrogen fueling stations may also be space limited. Thus, meeting specified separation distances may not always be possible for some hybrid and new hydrogen fueling stations. A facility operator may thus be required by the AHJ to address the risk implications of the exemption and include additional safety features or take other steps to address an increase in risk associated with the facility design and operation.

To ensure the safety of a hydrogen facility when compliance with all of the code requirements is not possible, a risk-informed permitting process could be used that includes consideration of risk insights as well as conformance with the other requirements in the relevant codes, standards, and regulations. Similarly, this process could be utilized for permitting unique or non-standard facility designs. In this permitting process, the facility operator may be required by the AHJ to perform a QRA of the proposed facility and include the results in the permit submittal. The QRA could be limited to show the impact of the exemption request. The inclusion of this risk information would allow the AHJs to make a risk-informed decision on whether to permit the facilities. However, it should be realized that AHJs generally do not have a clear understanding of the concept of risk and what are acceptable levels, and thus would require some level of training on the risk associated with hydrogen fueling facilities (this topic is addressed in the next section).

\subsection{RISK ASSESSMENT TOOLS FOR SUPPORTING THE PERMITTING PROCESS}

To facilitate the permitting of hydrogen fueling stations, the U.S. Department of Energy has launched an initiative to facilitate the permitting process for hydrogen fueling stations [13]. In brief, a key element of this initiative is a web-based toolkit that includes information fact sheets, networking charts to encourage information exchange among code officials who have gone or are going through the permitting of hydrogen fueling stations, templates to show whether a proposed station footprint conforms to requirements in the jurisdiction, and a compendium of requirements incorporated in key codes and standards. The toolkit will be augmented by workshops for code officials and station developers in jurisdictions that are likely to have hydrogen fueling stations in the near term.

A similar toolkit being developed within the Canadian Hydrogen Safety Program also includes a QRA tool that can be used by facility designers to evaluate the risk associated with their proposed facility design and operation, particularly when the facility is unique or does not meet the applicable codes and standards, and to identify alternative cost-effective methods to reduce the risk to acceptable levels [14]. Currently, the QRA features are limited to examining only a limited number of possible accident sequences.

The European Union Network of Excellence on Hydrogen Safety 'HySafe" has been developing risk assessment and risk communication tools for hydrogen applications for the past few years [15]. The 
Hydrogen Incident and Accident Database (HIAD) once developed will become an invaluable source of information for risk management decisions. A new HySafe initiative worth mentioning is HyQRA intended to achieve a better consistency in site risk assessment [16].

Development of comprehensive QRA models is required to support a risk-informed permitting process, including the development of risk-informed codes and standards. The QRA tools should be proactively used for AHJ education and training so that regulators can learn about potential consequences, frequencies/probabilities, and risk of selected failure scenarios related to likely station designs and associated technologies. The tool may also be used to help design engineers in understanding compliance with codes and standards, risks of typical component failures, and for developing adequate mitigation strategies.

The QRA toolkit should include the capability to quantify the risk models and evaluate parameter importance in order to illustrate which events are most important to facility risk. In addition, the capability to propagate parameter and consequence uncertainty distributions through the QRA models is desirable in order to identify the associated uncertainty in the resulting risk values. Finally, the capability to perform sensitivity studies to evaluate the importance of key assumptions and modeling uncertainties and to perform alternate design evaluations should be available. A desirable feature of the alternate design evaluation capability would be the inclusion of a cost-benefit capability.

In summary, the QRA tool should include the following features:

- Simple but realistic consequence models for all possible accident types (i.e., jet fires, vapor cloud explosions, flash fires, pool fires, etc.). Alternatively, it may be possible to limit the modeling to address just the dominant accident types.

- Generic event tree models delineating the sequence of events for each possible accident initiator that can be internally manipulated to account for site-specific features.

- Fault trees to evaluate accident initiator frequencies and mitigation failure events represented in the event trees.

- Accepted data values and distributions for leak frequencies, component failure probabilities, human error rates, and phenomenological event probabilities (e.g., spontaneous ignition probability).

- Propagation of parameter uncertainty through the QRA models and performance of sensitivity studies to address assumptions and model uncertainties.

- Choice of risk criteria for establishing adequacy of the design (e.g., using the frequency of an individual injury or fatality; use societal risk approaches such as a Frequency-Number curve; address worker and user risk; and allows them to use the mean, median, or a selected percentile from the calculated risk distribution to make their decision).

The IEA Task 19 Hydrogen Safety group as part of its scope for the next 3 years will strive to develop a suite of realistic consequence models for all possible types of accidents as well as to establish the framework to achieve consistency in site risk assessment taking into consideration the HySafe HyQRA approach. The IEA Task 19 Hydrogen Safety group will strive to develop a compendium of QRA modeling methods and data in order to identify gaps.

\subsection{SUMMARY}

Establishment of risk criteria is a key element required to utilize a risk-informed approach to the development of hydrogen codes and standards. The necessity to comply with risk acceptance criteria dictates that: a) any product must have a basic design that satisfies risk acceptance criteria and thus ensures a minimum acceptable level of safety under intended operating conditions; b) methods and 
tools are required to measure and verify compliance with acceptable levels of risk and c) codes and standards that identify minimum design, performance and installation requirements as well as regulations that guide the permitting and approval process have to reflect those risk acceptance criteria in order to become risk-informed.

There is a substantial amount of data and various approaches to selection of risk acceptance criteria. IEA Task 19 within its subtask on risk management intends to perform detailed analyses of similar data available within IEA countries and recommend uniform risk acceptance criteria for hydrogen refueling infrastructure for implementation globally.

Once acceptable levels of risk are determined they can be incorporated into hydrogen codes and standards thus making them risk-informed. Compliance with risk-informed codes and standards would mean that station designs meeting their requirements automatically meet acceptable levels of safety and can be permitted without additional requirements.

A risk-informed process utilizes risk insights obtained from QRAs combined with other considerations to establish code requirements. The QRAs are used to identify and quantify scenarios for the unintended release of hydrogen, identify the significant risk contributors at different types of hydrogen facilities, and to identify potential accident prevention and mitigation strategies to reduce the risk to acceptable levels. Examples of other considerations used in this risk-informed process can include the results of deterministic analyses of selected accidents scenarios, the need for defense-in-depth for certain safety features (e.g., overpressure protection), the use of safety margins in the design of highpressure components, and requirements identified from the actual occurrences at hydrogen facilities. A key component of this process is that both accident prevention and mitigation features are included in the code and standard requirements.

QRA can be used in several different ways to help establish the code and standard requirements for hydrogen facilities. First, by analyzing a complete set of possible accidents, the risk drivers for these facilities can be identified. Accident prevention and mitigation features can then be specified to address all accidents. The number or type of specified requirements can be determined by both the cause and frequency of the accident and the associated risk.

QRAs can also evaluate the risk associated with selected accidents used in establishing code requirements. An example of this is the selection of a particular leak size to establish safety distances. Safety distances based on a probable leak diameter can introduce a high level of risk from leaks that are less probable but which result in high consequences. Thus, it is desirable that risk considerations be included in the determination of accidents.

The challenge faced in permitting hydrogen fueling stations is to have a process that is relatively simple and fast but also ensures that the facilities are safe. To facilitate the permitting of hydrogen fueling stations, several software packages have been proposed and are under development in the U.S., Canada, and within the European Network of Excellence on Hydrogen Safety (HySafe).

The IEA Task 19 Hydrogen Safety group will strive to develop a compendium of QRA modeling methods and data in order to identify gaps as well as a suite of realistic consequence models for all possible types of accidents as well as to establish the framework to achieve consistency in site risk assessment.

\section{ACKNOWLEDGEMENTS}

The authors would like to thank US Department of Energy (US DOE) and Natural Resources Canada (NRCan) for their contributions to funding of this work. The authors would also like to thank Mr. Bill Hoagland, the Operating Agent of IEA Task 19 Hydrogen Safety, for his contribution to this paper. 


\section{REFERENCES}

1. ISO/IEC Guide 51: 1999, Safety aspects - Guidelines for their inclusion in standards

2. ISO/IEC Guide 73: 2002, Risk management - Guidelines for use in standards

3. "Injury Facts," 2007 Edition, National Safety Council.

4. "Responsible risks, safe space", Advisory Note from VROM Council and Council for Transport, Public Works and Water Management, June 2003, p. 61.

5. Determination of Safety Distances, European Industrial Gas Association, IGC Doc 75/07/E/.

6. "Risk Acceptance Criteria for Hydrogen Refueling Stations," European Integrated Hydrogen Project Phase 2, February 2003.

7. "Fires in or at Service Stations and Motor Vehicle Repair and Paint Shops," National Fire Protection Association, April 2002.

8. Proposals for revised policies to address societal risk around onshore non-nuclear major hazard installations. Initial regulatory impact assessment, HSE, 2007.

9. Chain studies: ammonia, chlorine and LPG. KPMG, TNO and ECORYS, The Netherlands, November 2004.

10. NFPA 55, "Standard for the Storage, Use, and Handling of Compressed Gases, and Cryogenic Fluids in Portable and Stationary Containers, Cylinders, and Tanks," 2005 Edition, National Fire Protection Association.

11. NFPA 52, "Vehicular Fuel Systems Code", 2006 Edition, National Fire Protections Association.

12. ISO/PDTS 20012 Gaseous hydrogen - Fuelling stations. ISO/TC 197 Working Group (WG) 11, Draft Technical Specification, March 2007.

13. Ohi, Jim, "Hydrogen Safety and Permitting Hydrogen Fueling Stations," to be presented at the $2^{\text {nd }}$ International Conference on Hydrogen Safety, San Sebastian, Spain, September 2007.

14. Hay, D. Robert, et al., "Hydrogen Safety, Training and Risk Assessment System," to be presented at the $2^{\text {nd }}$ International Conference on Hydrogen Safety, San Sebastian, Spain, September 2007.

15. Funnemark E., Engebo, A. "Development of tools for risk assessment and risk communication for hydrogen applications." Proceedings of the $1^{\text {st }}$ International Conference on Hydrogen Safety, Pisa, Italy, September 2005.

16. HySafe Joint Plan of Activities for months from 37 to 54, March 2007. 